\title{
Effective Field Theories, Reductionism and Scientific Explanation
}

\section{Stephan Hartmann*}

\begin{abstract}
Effective field theories have been a very popular tool in quantum physics for almost two decades. And there are good reasons for this. I will argue that effective field theories share many of the advantages of both fundamental theories and phenomenological models, while avoiding their respective shortcomings. They are, for example, flexible enough to cover a wide range of phenomena, and concrete enough to provide a detailed story of the specific mechanisms at work at a given energy scale. So will all of physics eventually converge on effective field theories? This paper argues that good scientific research can be characterised by a fruitful interaction between fundamental theories, phenomenological models and effective field theories. All of them have their appropriate functions in the research process, and all of them are indispensable. They complement each other and hang together in a coherent way which I shall characterise in some detail. To illustrate all this I will present a case study from nuclear and particle physics. The resulting view about scientific theorising is inherently pluralistic, and has implications for the debates about reductionism and scientific explanation.
\end{abstract}

Keywords: Effective Field Theory; Quantum Field Theory; Renormalisation; Reductionism; Explanation; Pluralism.

*Center for Philosophy of Science, University of Pittsburgh, 817 Cathedral of Learning, Pittsburgh, PA 15260, USA (e-mail: shart@pitt.edu) (correspondence address); and Sektion Physik, Universität München, Theresienstr. 37, 80333 München, Germany. 


\section{Introduction}

There is little doubt that effective field theories are nowadays a very popular tool in quantum physics. They are almost everywhere, and everything is considered to be an effective field theory (EFT). Particle physicists, for example, even take a supposed-tobe fundamental theory such as the celebrated Standard Model of the electromagnetic, weak, and strong interactions to be an EFT (Meissner, 1992). Nuclear physicists systematically derive low-energy EFTs from quantum chromodynamics (a theory which is part of the Standard Model) to account for the dynamics of protons and neutrons in atomic nuclei at low and intermediate energies (Van Kolck, 1999). And solid state theorists formulate age-old models such as the BCS theory of conventional superconductivity in the language of EFTs (Shankar, 1999). Even gravitational physicists seem to be infected by the EFT-virus: they consider the general theory of relativity to be the starting point of a power-series expansion, to which higher-order quantities that are still invariant under general coordinate transformations have to be added, to account for the physics at higher energies (Donoghue, 1994a,b). The resulting EFTs include quantum corrections to Einstein's theory which are considered to be footprints of a quantum theory of gravity, a theory we do not yet have, but which we might be able to find (or divine) by following the EFT programme.

EFTs account for the physics at a given energy scale by relying only on those entities which are relevant at that scale. These entities are, for example, quarks, leptons and the gauge bosons in the Standard Model, pions and nucleons in nuclear physics at not too high energies, and Cooper pairs in the theory of conventional superconductors. Using these effective degrees of freedom makes computations tractable and provides some intuitive understanding of what is physically going on at the energy scale under consideration. The resulting descriptions are very accurate; this indicates that the effects of the physics at higher energies do not really make a difference at lower scales in these cases: the physics at high energies is 'decoupled' from the physics at low energies. Its effects are contained in a few parameters of the low-energy theory. The formalism of EFTs makes all this more precise. Besides, there is a systematic and controlled way to derive low energy EFTs from a more fundamental high energy theory.

For a long time, the criterion of renormalisability was considered to be a sine qua non for any acceptable physical theory. After all, we want our theories to give finite results and if higher orders in a perturbation expansion diverge, the theory is in trouble. Renormalisation is a way to 'get rid' of these infinities, but it turns out that many EFTs cannot be renormalised and are therefore, according to the old view, in trouble. Their appraisal requires that we reconceptualise what renormalisation 
amounts to. This reconceptualisation took place in the 1970s; it is a consequence of a realistic (as opposed to a formalistic) interpretation of the cut-off parameter in quantum field theories and of the insights of renormalisation group theory.

Besides their value in research, EFTs also played a role in a recent debate among scientists which was, however, in the end mainly about funding issues. In this debate, particle physicists (most prominently Steven Weinberg) advocated building a Superconducting Super Collider (SSC), an extraordinarily expensive particle accelerator, which should help theorists find the 'final theory' (Weinberg, 1993). In the end, the US Congress did not make this dream come true. Weinberg's opponents, such as the solid state physicists Philip W. Anderson and James Krumhansl, argued convincingly against this project. Since he could not point to technological spin-offs, Weinberg's main argument for the SSC was the very foundational character of particle physics: 'Particle physics is in some sense more fundamental than other areas of physics' (1987, p. 434). It is more fundamental because it is 'on a level closer to the source of the arrows of explanation than other areas of physics' (ibid., p. 437). Anti-reductionists, on the other hand, point to the autonomy of the different levels of organisation. All these levels have their own ontology and their own laws, so why not call them fundamental as well? ${ }^{1}$ It is not an easy task to make more precise what it means exactly that different levels of organisation are autonomous. However, within the programme of EFTs, the notion of quasi-autonomy can be given a precise meaning and the relation of one level of organisation to a deeper level can be studied. We will come back to this issue below and discuss its consequences for the reductionism debate.

Despite the great importance of EFTs in actual scientific practice and in an important debate among scientists, philosophers of science have not paid much attention to EFTs. Following a seminal (though philosophically controversial) paper by Cao and Schweber (1993), some articles have been published which mainly focus on the issue of renormalisation and on the role of the renormalisation group. ${ }^{2}$ In 1996, a remarkable conference on the conceptual foundations of quantum field theory took place at Boston University. Its participants included many of the main contributors to the development of quantum field theory and to the EFT programme. ${ }^{3}$ At this conference a lot of attention was paid to EFTs. A full philosophical appraisal of EFTs and their consequences is still missing however. This is the aim of this article. Philosophers of science have discussed theories and models a great deal. EFTs

\footnotetext{
${ }^{1}$ This debate is carefully reconstructed in Cat (1998).

${ }^{2}$ See the articles by Huggett and Weingard (1995), Robinson (1992), Cao (1993), Schweber (1993a), and the more general articles by Schweber (1993b, 1995).

${ }^{3}$ The proceedings of this conference are published in Cao (1999).
} 
share similarities with both of them. My first goal will therefore be to locate EFTs in the 'conceptual space' defined by these tools. I will do this by looking at the functions of theories, models, and EFTs in the research process and conclude that EFTs share many of the functions of theories and models. Theories and models are, however, also an indispensible tool of scientific research and I will defend a pluralistic account of scientific theorising on the basis of a detailed case study. My second goal is then to draw some more general conclusions from my reconstruction of scientific practice, namely about the issues of reductionism and scientific explanation.

The remainder of this article is organised as follows. Section 2 provides some historical background and introduces the concept of EFTs. Section 3 points out the functions of, and relations between, theories, models, and EFTs on the basis of a case study from nuclear and particle physics. Philosophical conclusions concerning pluralism, reductionism and scientific explanation are then drawn in Section 4. Finally, Section 5 summarises my main points.

\section{The Emergence of Effective Field Theories}

Although the first paper on EFTs appeared only in 1979 (Weinberg 1979, 1980b), the general idea behind it is much older. As early as in 1936, the German physicists Hans Euler and Werner Heisenberg calculated the process of photon-photon scattering at small photon energies within the framework of the quantum theory of fields developed by Paul Dirac a couple of years earlier. Euler and Heisenberg derived a non-linear modification of Maxwell's equations which could however be interpreted in an intuitive way. Another early example of an EFT is Fermi's theory of weak interactions. Both theories will be discussed in Section 2.1. For a long time, however, theories such as the ones by Euler, Heisenberg and Fermi were not taken seriously because they were not renormalisable. Only after a 'change in attitude' (Weinberg) among physicists - mainly due to the development of renormalisation group techniques - was it possible to consider non-renormalisable theories as full-blown scientific achievements. To arrive at the current conception of EFTs, one more step was required. In 1975, Appelquist and Carazzone derived a theorem according to which under certain conditions the heavy particles in a theory decouple from the low-energy physics (modulo a renormalisation of the parameters of that theory). I will sketch these developments in Section 2.2. Finally, I present two ways of applying EFTs, viz. the bottom-up approach and the top-down approach, both of which have a variety of applications in physics. 


\subsection{Two early examples}

This section introduces two early examples of an EFT, the Euler-Heisenberg theory of photon-photon scattering (Section 2.1.1) and the Fermi theory of weak interactions (Section 2.1.2). Both theories exhibit typical features of an EFT which are compiled in Section 2.1.3.

\subsubsection{The Euler-Heisenberg theory}

Soon after Dirac presented his first attempts towards a quantum theory of fields, Euler and Heisenberg applied this theory to the process of photon-photon scattering. ${ }^{4}$ The authors did not worry much about the fact that Dirac's theory had various conceptual problems at that time. Quite to the contrary, by working out interesting applications of the theory and by exploring its consequences, Euler and Heisenberg hoped to get a hint in which direction one has to look in order to find a satisfactory quantum theory of fields. In a letter to Wolfgang Pauli, Heisenberg wrote about a similar situation a couple of months earlier:

In respect to quantum electrodynamics, we are now in the same state as we were in 1922 in respect to quantum mechanics. We know that everything is wrong. But in order to find the direction in which we have to depart from the present state, we have to know much better the consequences of the present formalism. ${ }^{5}$

Following this line of thought, the motivation of Euler and Heisenberg's joint work was to get an understanding of the consequences of Dirac's provisional formalism in order to find ways to improve it.

Photon-photon scattering is a typical quantum electrodynamical process which has no classical analogue. It does not occur in classical physics because of the linearity of Maxwell's equations ('superposition principle'). In quantum electrodynamics, however, the superposition principle does not hold. Now, photons can interact and the elementary process, for the discovery of which Euler attributes to Otto Halpern and Peter Debye, is this: the two photons scatter and create an electron-positron pair which then decays back into two photons, respecting energy and momentum conservation. This effect will lead to a modification of Maxwell's equations for the vacuum by adding non-linear terms to it.

\footnotetext{
${ }^{4}$ The first paper on this subject matter was published by Euler (1936); Heisenberg and Euler (1936) contains a considerable simplification and generaliation of Euler's calculation.

${ }^{5}$ Letter to W. Pauli dated 25 April 1935; quoted from Cassidy (1995, p. 416), my translation.
} 


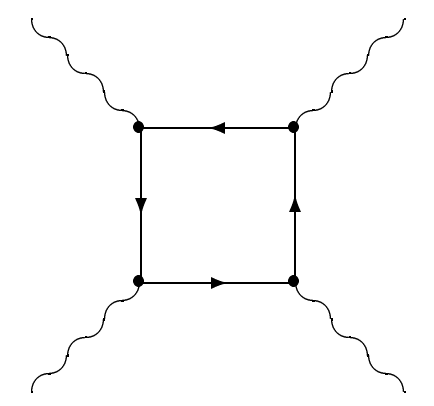

Figure 1: Feynman diagram for photon-photon scattering.

Euler and Heisenberg did not tackle the full problem but considered a special case. While Breit and Wheeler calculated the cross section for this process for high photon energies, in which real electrons and positrons are created, Euler and Heisenberg's attention focused on photons with energies well below the production threshold of electrons and positrons.

But even this is not an easy problem. In the modern language of Feynman diagrams, the "box-diagram" depicted in Fig. 1 has to be calculated. ${ }^{6}$ Euler and Heisenberg, of course, did not know Feynman's efficient methods, but they calculated essentially this diagram and so we use it here to visualise the corresponding elementary process. Since the process is of the fourth order, it is clear that there are considerable mathematical difficulties which show up when calculating the transition amplitude. These difficulties even show up when Feynman diagrams are used explicitly. ${ }^{7}$

For the details of the calculation we now follow the modern reconstruction given by Itzykson and Zuber (1980, pp. 195f). This work is focused on Euler and Heisenberg (1936), which is a simplification and generalisation of Euler (1936). The modern covariant formulation goes back to Schwinger (1973, pp. 123-134). If one requires (1) U(1) gauge invariance, (2) Lorentz invariance and (3) parity invariance, any Lagrangian density which should account for the process of photon-photon scattering

\footnotetext{
${ }^{6}$ Besides this diagram, there are two other diagrams which contribute in the same (fourth) order in perturbation theory; they are obtained by permutating the external photon lines, cf. Jauch and Rohrlich (1976, Ch. 13).

${ }^{7}$ For an exact calculation, see Jauch and Rohrlich (1976, Ch. 13).
} 
must have the following structure:

$$
\mathcal{L}_{e f f}=-\frac{1}{4} F^{\mu \nu} F_{\mu \nu}+\frac{a}{m_{e}^{4}}\left(F^{\mu \nu} F_{\mu \nu}\right)^{2}+\frac{b}{m_{e}^{4}} F^{\mu \nu} F_{\nu \sigma} F^{\sigma \rho} F_{\rho \mu}+\mathcal{O}\left(F^{6} / m_{e}^{8}\right)
$$

Here $m_{e}$ is the mass of the electron, and $F^{\mu \nu}$ is the field-strength tensor of the electromagnetic field. $a$ and $b$ are dimensionless constants which have to be determined.

Note that there are no electron degrees of freedom in Eqn (1). This is not necessary, however, since the considered process is purely photonic. Electrons do not show up explicitly. The first term in Eqn (1) is the well-known contribution of 'free' photons. All other terms are part of a systematic expansion in $1 / m_{e}$, respecting the symmetries mentioned above. The non-linearity of these terms reflects the violation of the superposition principle. For low photon energies $\left(E_{\gamma} \ll m_{e}\right)$, it suffices to consider only the first three terms in this expansion.

All information about this energy regime is therefore contained in the constants $a$ and $b$. But how can these constants be determined? One possibility is to do the explicit expansion of the original Lagrangian density of quantum electrodynamics by 'integrating' out the electron degrees of freedom. There are efficient calculational tools available now which, however, presuppose the path-integral formulation which was not available when Euler and Heisenberg performed the calculations. Instead, Heisenberg and Euler (1936) applied elegant mathematical technique which essentially led to the same result. However, in the original publication, Euler (1936) chose another way. He calculated a special case of the process under consideration in two ways: exactly and by using the effective Lagrangian density given by $\mathcal{L}_{\text {eff }}$. Comparing both results, he obtained the following values for the two constants:

$$
a=-\frac{\alpha_{0}^{2}}{36}, \quad b=\frac{7 \alpha_{0}^{2}}{90}
$$

with the fine-structure constant $\alpha_{0}=1 / 137$. In terms of the electric and magnetic field strengths $(E$ and $B$ ), the resulting effective Lagrangian density has the following form:

$$
\mathcal{L}_{\text {eff }}^{E H}=\frac{1}{2}\left(E^{2}-B^{2}\right)+\frac{2 \alpha_{0}^{2}}{45 m_{e}^{4}}\left[\left(E^{2}-B^{2}\right)^{2}+7(E \cdot B)^{2}\right] .
$$

This expression has been the basis of many subsequent calculations; it is still used today (see, for example, Becker, McIver and Schlicher (1989) for a quantum optical application).

\subsubsection{The Fermi theory}


Another historical example is Enrico Fermi's theory $(1933,1934)$ of weak interactions which was developed soon after Wolfgang Pauli suggested the existence of the neutrino as a way to account for the continuous beta spectra discovered by James Chadwick in 1914. These spectra gave rise to various speculations, including Niels Bohr's famous suggestion of giving up energy conservation in order to account for them.

Following the model of quantum electrodynamics, Fermi developed a theory which uses Pauli's hypothesis and describes the elementary process $n \rightarrow p+e^{-}+\bar{\nu}_{e}$ quantum field theoretically. In this reaction, a neutron $(n)$ decays in a proton $(p)$, an electron $\left(e^{-}\right)$and an electron anti-neutrino $\left(\bar{\nu}_{e}\right) .{ }^{8}$ Since there was nothing known about the details of the interaction, Fermi had to start from scratch, with some, but not many experimental constraints. He assumed that the interaction is pointlike and that the interaction Hamiltonian is given by the product of the operators representing the relevant particles multiplied by a coupling constant which has to be derived from experiment. This coupling constant has the dimension energy ${ }^{-2}$.

With these assumptions and the application of perturbation theory, Fermi was able to derive various mean lives of unstable nuclei as well as the shape of the electron spectra. Fermi's theory was highly successful and remained valid until experiments established that parity is violated in weak interactions. In order to account for this, Richard P. Feynman and Murray Gell-Mann (along with Robert E. Marshak and E.C.G. Sudarshan) suggested in 1958 a modification of Fermi's theory, the $V-A$ theory. This empirically very successful theory (apart from an explanation of CP violation) is still based on a point interaction and uses the same coupling constant Fermi used. Like Fermi's theory, the $V-A$ theory is not renormalisable.

\subsubsection{Some conclusions}

The theories of Fermi and Euler and Heisenberg have some interesting features. These features are typical for EFTs and can be summarised as follows:

1. Both theories take only the relevant fields into account.

These fields, called effective fields, are the photon field (represented by the electrical and magnetical field strengths) in the Euler-Heisenberg example, and the proton, neutron, electron and neutrino fields in the Fermi theory of weak interactions. Other fields, such as the electron field in the Euler-Heisenberg case, do not show up explicitly at the respective energy scale. Their presence is hidden, reflected by the non-linear terms in the effective Lagrangian density.

\footnotetext{
${ }^{8}$ Fermi took it to be a neutrino. He did not yet know about the conservation of lepton number and other kinds of neutrinos.
} 
2. Both theories are valid only at a given energy scale.

The derivation of the Euler-Heisenberg theory presupposes that the photon energy is small compared to the rest mass of the electron. Applications to higher energies are not justified. The Fermi theory violates unitarity at high energies (above $300 \mathrm{GeV}$ ) and is therefore also valid only at a specific energy scale. For higher energies, alternative theories are needed.

3. Both theories are non-renormalisable.

It can be shown on general grounds that the theories by Euler and Heisenberg and by Fermi are non-renormalisable. Divergent results show up once higher order contributions to the perturbation expansion are calculated. To eliminate them, a renormalisation scheme has to be specified. This renormalisation scheme is therefore part of the definition of the EFT if one is interested in higher order contributions.

4. Both theories are based on certain symmetries.

Symmetry requirements are very important in the construction process of an EFT. This is demonstrated by our reconstruction of the development of the Euler-Heisenberg theory; to get the effective Lagrangian density of Eqn (1), all possible terms with the required symmetries have to be included - whether they are renormalisable or not. The hard job is then to determine the coefficients of the respective terms in the expansion.

Symmetry considerations also played a role in the formulation of the Fermi theory. Since there was not much information about the structure of the weak interaction, simplicity suggested a scalar interaction term. After the discovery of the violation of parity conservation in the weak interactions, a combination of all other possible types of Lorentz-invariant pointlike interactions were tried. Fortunately, there are only five of them (scalar $(S)$, pseudoscalar $(P)$, vector $(V)$, axialvector $(A)$ and tensor $(T)$ interactions) and a set of crucial experiments finally selected the $V-A$ of Feynman and Gell-Mann as the only one compatible with available experimental data (see Franklin, 1990).

5. Both theories produce scientific understanding.

The work of Euler and Heisenberg had many motivations. Among those were the wish to apply, to test, and to find out the consequences of Dirac's provisional quantum theory of fields. Another motive was to get a tractable mathematical formalism which allowed the calculation of the interaction of photons at low 
energies. This goal suggested the chosen approximation scheme. Yet another motive was to get some intuitive understanding of the respective processes. This is directly substantiated by a section title in Euler's original paper (1936, p. 400; my translation):

$\S 1$. Provisional statement of an intuitive expression for the interaction $\bar{U}_{1}$ of light with light $[\ldots]$

In the course of this work Euler mentions several times that his aim is to derive an intuitive (anschaulich) expression which describes the physically relevant processes. What does this mean? It was already well known at the time that certain materials react in a non-linear way to external fields. The guiding idea for Euler and Heisenberg now was that even the vacuum exhibits such nonlinear behaviour. Starting from the quantum theory of electrons and photons, they succeeded in deriving non-linear corrections to Maxwell's equations for the vacuum based on this analogy. The corrections suggest the interpretation that even the vacuum can be polarised, an effect which is responsible for the non-vanishing photon-photon cross section. This analogy to an already wellunderstood effect guided their derivation and helped to interpret the final result. The resulting corrections to Maxwell's equations are also very easy to handle mathematically, once they are derived. It is a typical feature of EFTs that they are very easy to handle (compared to the full theory), and also produce (local) understanding (unlike the full theory, as I will argue below, see Section 3.1.1). Pragmatic and cognitive goals meet here in an interesting way.

The Fermi theory produces understanding in so far as it is the simplest modification of quantum electrodynamics which accounts for the phenomena of weak interactions.

In the years between the development of Euler, Heisenberg and Fermi's theories and the late nineteen-forties, theoretical research in quantum field theory focused mainly on formulating a theory which avoids the divergences in the perturbative expansion from which Dirac's theory suffered. Satisfactory covariant renormalisation schemes were finally introduced by Dyson, Feynman, Schwinger, and Tomonaga. ${ }^{9}$ Motivated by the astonishing success of QED, as manifested most convincingly in the precise calculation of the Lamb shift and the anomalous magnetic moment of the electron, renormalisability soon became the key criterion for the selection of quantum

\footnotetext{
${ }^{9}$ See Schweber (1994) for an historical account of these exciting developments.
} 
field theories for other phenomena (such as the weak and strong interactions). Henceforth, non-renormalisable theories had at best a provisional status: useful, perhaps, for various calculations, but of no deeper significance. This view began to crumble with the development of renormalization group techniques in the 1970s which finally led to the rehabilitation of non-renormalisable theories and the establishment of the research programme of EFTs. The next section will sketch this development in more detail.

\subsection{Renormalisation and the renormalisation group}

The modern development of EFTs is closely related to a new conceptualisation of renormalisation. This section will give a concise reconstruction of this development. It all began with quantum electrodynamics (QED) and the supposed need to find a way to eliminate the notorious infinities in the perturbation expansion. Let's first look at this expansion.

Let $H_{I}$ be the interaction Hamiltonian of a system and let $\Psi(t)$ be a field operator in the interaction picture. $\Psi(t)$ satisfies the Schrödinger equation

$$
H_{I} \Psi(t)=\mathrm{i} \frac{\partial \Psi(t)}{\partial t}
$$

which has the formal solution

$$
\Psi(t)=\Psi(-\infty)-\mathrm{i} \int_{-\infty}^{t} d t_{1} H_{I}\left(t_{1}\right) \Psi\left(t_{1}\right)
$$

In particle physics experiments, one is typically interested in the calculation of scattering processes. This only requires information about the asymptotic state $\Psi(+\infty)$ of the system under consideration. This state can be formally obtained from the initial state by applying the so-called $S$-matrix:

$$
\Psi(+\infty)=S \Psi(-\infty) .
$$

If $S$ is known, all relevant observables, such as scattering cross sections, can be obtained easily. More explicitly, $S$ is given by

$$
S=\sum_{n=0}^{\infty} \frac{(-\mathrm{i})^{n}}{n !} \int d^{4} x_{1} \ldots d^{4} x_{n} P\left\{H_{I}\left(x_{1}\right) \ldots H_{I}\left(x_{n}\right)\right\}
$$

with the time ordering operator $P$. Based on the last equation, a perturbation expansion can be derived which in turn can be translated into the language of Feynman 


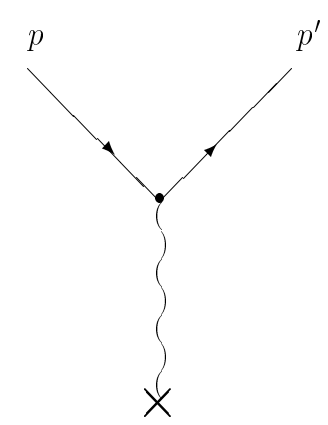

Figure 2: The main contribution to the potential scattering of an electron.

diagrams. These diagrams provide an intuitive identification of all contributions to $S$ with a representation of elementary spacetime processes which lead from the initial state to the final state while respecting all relevant conservation laws.

In quantum electrodynamics (QED), the theory we will focus on for a while, $S$ can be expanded in powers of the fine structure constant $\alpha_{0}: S=S^{(0)}+S^{(1)}+S^{(2)}+\ldots$, with $S^{(n)}$ being proportional to $\alpha_{0}^{n}$. Due to the smallness of $\alpha_{0} \approx 1 / 137$, a small number of terms suffices to determine $S$ with good accuracy.

But complications arise since divergences show up. Before pointing this out in some detail, a change of notation will be useful: Since $S=1$ describes the trivial reaction that the finite state is identical to the initial state, it is useful to introduce the so-called $T$-matrix which only captures non-trivial reactions: $S=1+\mathrm{i} T$.

\subsubsection{Renormalization in QED}

To present the idea of renormalisation, let us focus on one specific process and let us use the language of Feynman diagrams. ${ }^{10}$ The process we will focus on is the scattering of an electron by an external potential $A_{\text {ext }}$ (cf. Fig. 2). The first divergent term in the perturbation expansion of the $S$-matrix is depicted in Fig. 3. In this diagram, a photon is emitted by the 'incoming' electron and reabsorbed by the 'outgoing' electron. This photon is called virtual because it does not show up in the Feynman diagram as an external line with one loose end.

It is now interesting to ask what the energy and the momentum of this virtual photon are. Although energy and momentum conservation hold at the vertices, this

\footnotetext{
${ }^{10}$ I am following the clear presentation given by Lepage (1989). See also Mills (1993).
} 


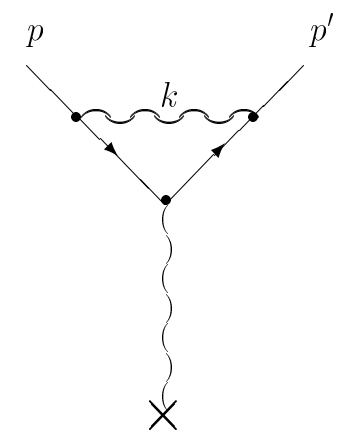

Figure 3: Vertex-correction to the potential scattering of an electron.

does not fix these values uniquely. A whole spectrum of values is possible, ranging from zero to infinity. In the mathematical formalism this means that all those contributions have to be added up, and here lies the source for the divergences.

In more technical terms, the story so far amounts to this: let $k$ be the 4-momentum of the virtual photon, and let $p$ be the momentum of the incoming electron with bare mass $m_{0}$ and bare charge $e_{0}$. 4-momentum conservation then requires that the momentum of the electron after the emission of the virtual photon is $p-k$. Similarly, if $p^{\prime}$ denotes the final electron 4-momentum, the 4-momentum of the electron immediately after the interaction with the external potential is then $p^{\prime}-k$. 4-momentum conservation does not give us any more information. $k$ is completely undetermined and all positive real numbers are possible for its absolute value. All resulting contributions then have to be integrated up to get the T-matrix. Applying the Feynman rules of QED, one obtains

$T^{(a)}=-e_{0}^{3} \int_{0}^{\infty} \frac{d^{4} k}{(2 \pi)^{4}} \frac{1}{k^{2}} \cdot \bar{u}\left(p^{\prime}\right) \gamma \frac{1}{\left(p^{\prime}-k\right) \cdot \gamma-m_{0}} A_{\text {ext }}\left(p^{\prime}-p\right) \cdot \gamma \frac{1}{(p-k) \cdot \gamma-m_{0}} \gamma u(p)$,

with spinors $u(p)$ and $\bar{u}\left(p^{\prime}\right)$ for the incoming and outgoing electron and the 4 -vector of Dirac's $\gamma$-matrices denoted by $\gamma$. This integral is divergent since the numerator is proportional to $k^{3} d k$, while the denominator is proportional to $k^{4}$ for large $k: T^{(a)}$ diverges logarithmically. Many other divergent terms like this show up in higher orders of the perturbation expansion, and many physicists came to the conclusion that this is an indication of a serious inconsistency in the very foundations of the theory. 
Pragmatically oriented as many physicists are, the empirical success of the renormalisation procedure developed around 1949 overruled these negative feelings. The suggested renormalisation schemes were not only a way to eliminate the infinities; they also led to new predictions and explanations of tiny corrections to observables. These quantities (such as the anomalous magnetic moment of the electron or the Lamb shift) have been measured in the laboratories with a remarkable accuracy. This is generally considered as an impressive confirmation of QED and the renormalisation scheme it applies. Let us see in some more detail how this works.

First, the divergent contributions have to be eliminated in all orders of perturbation theory. It turned out that in QED these terms have the same structure as terms which showed up already in the original Lagrangian density. Hence, they can be eliminated by a suitable redefinition of the parameters of the original Lagrangian density. These parameters are the mass and the charge of the electron:

$$
\begin{aligned}
m_{0} & \rightarrow m_{R}=m_{0}+\delta m \\
e_{0} & \rightarrow e_{R}=e_{0}+\delta e
\end{aligned}
$$

Here, $m_{R}$ is the renormalised mass and $\delta m$ is the modification of the mass due to higher order contributions to the perturbation theory. Simlilarly, $e_{R}$ is the renormalised charge and $\delta e$ includes the radiative corrections.

Dyson showed that a reparametrisation of this kind can be carried through in all orders of perturbation theory in QED. ${ }^{11}$ Now, while this procedure is so far purely mathematical and might look like a trick, a physical interpretation is required to justify this procedure. The basic idea behind this justification is to identify the renormalised mass and charge of the electron with its 'physical' (i.e. real) mass and charge. To get the observed finite values for these parameters, it has to be assumed that the bare mass and charge of an electron are also infinite, cancelling the infinite radiative corrections $\delta m$ and $\delta e$. In the absence of the radiation field, the mass as well as the charge of the electron would be infinite. It is the 'switching-on' of the radiation field which accounts for the finite mass and charge of the electron.

At first sight it might sound problematic to attribute an infinite bare mass and charge to the electron. Indeed, Richard Feynman meant exactly this when he claimed that in QED the real problems are swept under the rug. For Feynman it does not help to simply state that there is no real problem because a finite result can be obtained by subtracting one infinite number (the calculated radiative correction) from another infinite number (the - fortunately! - unobservable bare mass or charge).

\footnotetext{
${ }^{11}$ Later, Salam and Weinberg completed Dyson's original proof; cf. Cao (1993, pp. 42f).
} 
But the situation is more subtle than this suggests. Let us go back to the example of the electron interacting with an external potential. Eqn (8) represented the contribution of the Feynman diagram from Fig. 3 to the scattering amplitude. Instead of integrating over all $k$ up to infinity, let us first introduce an upper limit $\Lambda_{0}$ to this integral. Doing so can be understood as a purely formal trick to keep the integral well-defined and the numbers finite. The value of the original integral can then be obtained by performing the limit $\Lambda_{0} \rightarrow \infty$ at the end of the calculation.

Next, let us go one step further. We consider another theory which has a cut-off $\Lambda<k<\Lambda_{0}$ and ask how the original theory with cut-off $\Lambda_{0}$ has to be modified in order to produce the same results as the theory with cut-off $\Lambda$. It turns out that the following Lagrangian density has to be subtracted:

$T^{(a)}(k>\Lambda)=-e_{0}^{3} \int_{\Lambda}^{\Lambda_{0}} \frac{d^{4} k}{(2 \pi)^{4}} \frac{1}{k^{2}} \cdot \bar{u}\left(p^{\prime}\right) \gamma \frac{1}{\left(p^{\prime}-k\right) \cdot \gamma-m_{0}} A_{e x t}\left(p^{\prime}-p\right) \cdot \gamma \frac{1}{(p-k) \cdot \gamma-m_{0}} \gamma u(p)$

To proceed, let us assume that all masses and external momenta ( $p$ and $\left.p^{\prime}\right)$ are much smaller than $\Lambda$, so that the quantities $m_{0}, p$ and $p^{\prime}$ can be neglected in the integrand. One obtains:

$$
\begin{aligned}
T^{(a)}(k>\Lambda) & \approx-e_{0}^{3} \int_{\Lambda}^{\Lambda_{0}} \frac{d^{4} k}{(2 \pi)^{4}} \frac{1}{k^{2}} \bar{u}\left(p^{\prime}\right) \gamma \frac{k \cdot \gamma}{k^{2}} A_{\text {ext }}\left(p^{\prime}-p\right) \cdot \gamma \frac{k \cdot \gamma}{k^{2}} \gamma u(p) \\
& \approx-e_{0}^{3} \bar{u}\left(p^{\prime}\right) A_{\text {ext }}\left(p^{\prime}-p\right) \cdot \gamma u(p) \int_{\Lambda}^{\Lambda_{0}} \frac{d^{4} k}{(2 \pi)^{4}} \frac{1}{\left(k^{2}\right)^{2}} .
\end{aligned}
$$

In order to get the full $T$-matrix in this order of perturbation theory, other diagrams have to be included as well. Treating them in the same way as described above, the following result obtains for that part of the scattering amplitude which can be neglected due to the introduction of the new cutoff $\Lambda$ :

$$
T(k>\Lambda) \approx-\mathrm{i} e_{0} c_{0}\left(\lambda / \Lambda_{0}\right) \bar{u}\left(p^{\prime}\right) A_{e x t}\left(p^{\prime}-p\right) \cdot \gamma u(p)
$$

with a dimensionless quantity $c_{0}$, which only depends on the ratio $\Lambda / \Lambda_{0}$ :

$$
c_{0}\left(\Lambda / \Lambda_{0}\right)=-\frac{\alpha_{0}}{6 \pi} \log \left(\Lambda / \Lambda_{0}\right) \text {. }
$$

Note that $\Lambda$ and $\Lambda_{0}$ are the only energy scales at high energies for this problem since $p, p^{\prime}$ and $m_{0}$ have been neglected.

Now $T(k>\Lambda)$ is certainly an important contribution to the scattering amplitude which cannot be ignored. However, it is possible to 'simulate' the contribution of this term in the Lagrangian density with cut-off $\Lambda$ by adding a counter term of the form:

$$
\delta \mathcal{L}_{0}=-e_{0} c_{0}\left(\Lambda / \Lambda_{0}\right) \bar{\psi} A \cdot \gamma \psi
$$


Astonishingly, $\delta \mathcal{L}_{0}$ has the same structure as the current-field coupling term in the original Lagrangian density of QED. The effect of the additional term can therefore be included by a redefinition of the charge parameter: $e_{0} \rightarrow e_{0}\left[1-c_{0}\left(\Lambda / \Lambda_{0}\right)\right]$.

To sum up: in a renormalisable quantum field theory such as QED, the contributions of the high-energy sector of the theory can be effectively taken into account by a reparametrisation of the original theory. As the above derivation shows, this procedure does not presuppose that the original cut-off $\Lambda_{0}$ goes to infinity. However, if one calculates this limit one is bound to assume that the bare mass and charge are indeed infinite in order to account for the finite values of various quantities which we obtain in experiments.

A theory is called renormalisable if such an absorption of the divergent contributions of the high-energy sector of the theory in the mass and charge parameter(s) can be accomplished at all orders in perturbation theory. Doing so is, however, not possible for all quantum field theories. In fact, only a very small subclass of all quantum field theories is renormalisable. Motivated by the enormous empirical success of QED, renormalisability soon became the selection criterion for the construction of new quantum field theories. In his speech during the award of the Nobel price for 1979, Steven Weinberg argued this point:

To a remarkable degree, our present detailed theories of elementary particle interactions can be understood deductively, as consequences of symmetry principles and of the principle of renormalizablity which is invoked to deal with the infinities (Weinberg, 1980a, p. 515).

Somewhat later in this speech, Weinberg addressed the issue of the role of renormalisability for his own work:

I learned about renormalization as a graduate student, mostly by reading Dyson's papers. From the beginning it seemed to me to be a wonderful thing that very few quantum field theories are renormalizable. Limitations of this sort are, after all what we most want; not mathematical methods which can make sense out of an infinite variety of physically irrelevant theories, but methods which carry constraints, because constraints can point the way towards the one true theory [.... At the time] I thought that renormalizability might be the key criterion, which also in a more general context would impose a precise kind of simplicity on our theories and help us pick out the one true physical theory out of the infinite variety of conceivable quantum theories [...]. I would say this a bit differently today, but I am more convinced than ever that the use of renormalizability as a 
constraint on our theories of the observed interactions is a good strategy (Weinberg, 1980a, p. 517).

Renormalisation started as a pragmatic scheme which allowed efficient and precise predictions but which was theoretically considered to be unsatisfying and perhaps only provisional. It soon became the selection criterion (besides symmetry principles) for future quantum field theories. The theories found in this way (such as the Standard Model) turned out to be highly successful. The subsequent developments, summarised in the next section, led to a rehabilitation of non-renormalisable theories. Renormalisation is not any longer considered to be a decisive criterion for theory choice, but one should not forget to address the issue why renormalisable theories such as the Standard Model are so successful. We shall come back to this below.

\subsubsection{A new conceptualisation of renormalisation}

Let us summarise the renormalisation story told so far: In a renormalisable theory, the divergent terms in the perturbation can be eliminated by redefining the parameters of the original theory. The standard reasoning for this is that the bare masses and charges of the particles involved are not observable because of the ineliminable presence of radiation fields. These parameters can therefore be chosen freely, e.g. one can choose them to be infinite. The observed values of the masses and charges are then due to compensation effects of the radiative corrections.

This story did not convince everyone. One of the most severe critics was Paul Dirac, the founder of the old quantum theory of fields in which the problem with infinities first occurred. Several year later he wrote:

This [i. e. the renormalisation programme] is quite nonsense physically, and I have always been opposed to it. It is just a rule of thumb that gives results (Dirac, 1983, p. 55). ${ }^{12}$

However amazed by the empirical success of the renormalisation machinery and the possibilities it opened up of constructing new theories such as the Standard Model, most physicists did not follow Dirac's skepticism. Those who did, such as the advocates of the programme of Axiomatic QFT, did not succeed in finding the supposed inconsistency in the foundations of the theory. ${ }^{13}$ The historical development took a different path. The key to make sense of renormalisation came from within physics and the guiding idea was to interpret realistically the cut-off parameter which

\footnotetext{
${ }^{12}$ See also Kragh (1990, pp. 165f).

${ }^{13} \mathrm{Cf}$. Wightman (1986) for a discussion of renormalisation in this programme. See also Cao (1997, pp. 217-219).
} 
showed up in renormalisation schemes. It represents the energy scale up to which the theory in question is applicable.

To see this, let us go back to our example. It turned out to be helpful to replace the upper limit of the integral by some cut-off $\Lambda$. The standard procedure, first suggested by Feynman, is to take the limit and let $\Lambda$ go to infinity at the end of the calculation. For Feynman, the cut-off was a purely formal calculational device ('formal interpretation'), and indeed there was a good reason for this, since a realistic interpretation of the cut-off leads immediately to a new problem.

In this case, photons with all energies contribute, for example, to the diagram of Fig. 3. Now, photons and electrons are not the only particles present at high energies. And these other particles (such as protons, muons and pions) also couple to the electron and the photon. If one interprets the cut-off parameter realistically, there is no reason why these particles and their interactions should not be included in the calculation. But in that case, maybe it is just these contributions that make the theory finite. According to the realistic interpretation of the cut-off (if taken in the limit $\Lambda \rightarrow \infty$ ), only the final theory, if there is any, should be finite. So there is no reason anymore why QED with photons and electrons should be renormalisable.

But there is another way to interpret this situation. One can say that there is no reason anymore to consider theories with a finite cut-off as second rank if the cut-off parameter is interpreted realistically. This parameter then just reflects the production threshold of new particles (muons, pions, etc.) and the theory is henceforth only applicable up to this energy. A realistic interpretation of the cut-off leads to a rehabilitation of theories with a finite cut-off.

The formalism sketched so far can be easily extended to solve the following problem: given a theory with cut-off $\Lambda_{1}$, how can we get from there to a theory with a higher cut-off $\Lambda_{2}$ ? If no new particles show up in the energy regime between $\Lambda_{1}$ and $\Lambda_{2}$, it turns out that only the parameters of the theory (masses, charges) have to be changed ('renormalised'). Consequently, the masses and charges of particles depend on the energy scale under consideration; they have no absolute values. To get the values of these parameters on a higher or lower scale, the so-called renormalisation group equations have to be solved ${ }^{14}$.

Let us again illustrate this with our example from QED. As we saw, the elimination of states above some energy $\Lambda_{0}<\Lambda$ (with $\Lambda$ being the cut-off of the original theory) can be obtained by adding additional local terms of the form

$$
\delta \mathcal{L}=-e_{0} c_{0}\left(\frac{\Lambda}{\Lambda_{0}}\right) \bar{\psi} \gamma_{\mu} A^{\mu} \psi-m_{0} \tilde{c}_{0}\left(\frac{\Lambda}{\Lambda_{0}}\right) \bar{\psi} \psi,
$$

\footnotetext{
${ }^{14}$ See Fischer (1999) for a general introduction.
} 
with dimensionless parameters $c_{0}$ und $\tilde{c}_{0}$ proportional to $\ln \Lambda / \Lambda_{0}$. The new Lagrangian density is then

$$
\mathcal{L}^{\prime}=\mathcal{L}_{Q E D}+\delta \mathcal{L}=\bar{\psi}\left(\mathrm{i} \gamma_{\mu} \partial^{\mu}-e_{\Lambda} \gamma_{\mu} A^{\mu}-m_{\Lambda}\right) \psi,
$$

with the renormalised charge and mass given by

$$
\begin{aligned}
e_{\Lambda} & =e_{0}\left[1+c_{0}\left(\frac{\Lambda}{\Lambda_{0}}\right)\right], \\
m_{\Lambda} & =m_{0}\left[1+\tilde{c}_{0}\left(\frac{\Lambda}{\Lambda_{0}}\right)\right] .
\end{aligned}
$$

Changing the cut-off can therefore be compensated by changing the parameters of the theory. It can be shown that the parameters obey the renormalisation group equations:

$$
\begin{aligned}
\Lambda \frac{d e_{\Lambda}}{d \Lambda} & =\beta\left(e_{\Lambda}\right) \\
\Lambda \frac{d m_{\Lambda}}{d \Lambda} & =m_{\Lambda} \gamma\left(e_{\Lambda}\right),
\end{aligned}
$$

with appropriate functions $\beta$ and $\gamma$.

These equations grew out of the research of Michael Fisher, Leo Kadanoff and most importantly Kenneth Wilson in the context of solid state physics. It happened to have fruitful applications in particle physics as well. Here is the upshot of all this: (1) interpreting the cut-off realistically leads to a reappraisal of theories with a finite cut-off. (2) There is a systematic algorithm as to how to change the parameters of a theory when the energy scale is changed. (3) This algorithm, introduced here only for the case of a specific renormalisable theory, can be extended to other quantum field theories, including non-renormalisable quantum field theories, and to applications where new particles show up in the energy regime between the two cut-offs.

\subsubsection{The decoupling theorem}

Only one more idea is missing to make EFTs a powerful tool: the decoupling theorem, proved by Appelquist and Carazzone in 1975. In its simplest case, this theorem demonstrates that for two coupled systems with different energy scales $m_{1}$ and $m_{2}$ (with $m_{2}>m_{1}$ ) and described by a renormalisable theory, there is always a renormalisation condition according to which the effects of the physics at scale $m_{2}$ can be effectively included in the theory with the smaller scale $m_{1}$ by changing the parameters of the corresponding theory. The decoupling theorem implies the existence of 
an EFT at scale $m_{1}$ which will, however, cease to be applicable once the energy gets close to $m_{2}$.

One might think that this is not such a spectacular result since the whole edifice of physics is grounded on the assumption that empirical reality is layered so that for the physics at a given energy scale the details of the physics at much higher energies do not really matter. The idea of eliminating the physics at higher energies to get an effective account that is valid only at lower energies is also quite popular in other parts of physics. In his beautiful book Qualitative Methods in Quantum Theory, Migdal (1977) discusses an instructive example from quantum mechanics. Let $S$ be a system which is composed of a fast subsystem $S_{f}$ and a slow subsystem $S_{s}$, characterised by two frequencies $\omega_{f}$ and $\omega_{s}$. It can be shown that the effects of $S_{f}$ on $S_{s}$ can be taken into account effectively by adding a potential energy term to the Hamiltonian operator of $S_{s}$. In this case, as well as in many other cases, one ends up with an effective Hamiltonian operator for the subsystem characterised by the smaller frequency (or energy). It is interesting, however, that the decoupling theorem holds, given certain assumptions, also in quantum field theory. This is far from trivial if one recalls all those complicated radiative corrections which have to be taken into account here.

The decoupling theorem gives further legitimacy to non-renormalisable theories. If that theorem holds, the physics at higher energies can be effectively included in the parameters of a non-renormalisable EFT. Higher energy scales decouple and empirical reality seems to be divided into a set of 'quasi-autonomous domains', each theoretically captured by an EFT which employs only those particles and their interactions that are relevant at that scale. The domains are only quasi-autonomous since the effects of the physics at higher energy scales get more important once the energy reaches the cut-off energy of the EFT under consideration. It should be noted that EFTs can also be divined or obtained if one is not in the possession of a fundamental and renormalisable theory such as QED. It might, however, be more difficult to 'anchor' these theories, as the example of Fermi's theory showed. In these cases, finding a suitable EFT is more like guessing.

Does the decoupling theorem imply that empirical reality is, as a whole, layered into quasi-autonomous domains, as suggested by some authors? No, since the decoupling theorem is based on assumptions which may not always be fulfilled. Most importantly, the decoupling theorem (as proved by Appelquist and Carazzone) presupposes that there is a renormalisable theory of the composite system which is the starting point of the decoupling procedure. Without such a theory, which is supposed to be valid on all energy scales, the decoupling theorem cannot be applied. Furthermore, the decoupling theorem presupposes that different mass scales exist in 
the underlying renormalisable theory. But sometimes mass scales do not separate neatly, as examples from the theory of complex systems (such as turbulence) demonstrate. In these cases the physics at high energies cannot simply be absorbed in the parameters of a low-energy theory and the picture of empirical reality as layered into a hierachy of quasi-autonomous domains turns out to be too wild an extrapolation.

\subsection{Two ways to apply EFTs}

There are two ways to use EFTs in physics, the bottom-up approach and the top-down approach. I will describe both of them in some detail.

\subsubsection{The bottom-up approach}

This strategy is closely related to observable phenomena and some think that this is the way physics has to proceed. We will look at these arguments below (Section 4.2).

To apply this strategy, two scenarios have to be distinguished. First, there might be no relevant theory at all. In this case one has to start from scratch and construct a Lagrangian density from the particles, symmetries and interactions assumed to be relevant at the energy scale under consideration. Second, there is already some EFT $T_{1}$ which represents the physics at some energy scale, characterised by a cut-off parameter $\Lambda_{1}$. This theory might be, for example, QED or the Standard Model, both of which are - despite being renormalisable - considered to be EFTs. They might be applicable only up to some maximal energy $\Lambda_{1}$. At higher energies, new phenomena might happen to show up, and $T_{1}$ does not account for them. In order to obtain a new theory $T_{2}$ (valid up to some energy $\Lambda_{2}>\Lambda_{1}$ ) from the old theory $T_{1}$, two more cases have to be distinguished:

1. There are no new particles between $\Lambda_{1}$ and $\Lambda_{2}$.

In this case all the parameters of $T_{1}$ (i.e. charges and masses) have to be modified according to the renormalisation group equations. If the energy is less than $\Lambda_{1}$, both theories will give the same results for observable phenomena. But $T_{2}$ can also be applied for the energy range between $\Lambda_{1}$ and $\Lambda_{2}$. It should be noted that the relation between $T_{1}$ and $T_{2}$ for energies up to $\Lambda_{1}$ is very interesting. On the one hand, the theories differ from each other because their respective mass and charge parameters have different numerical values, while on the other hand, both are empirically equivalent.

Even if there are no new particles in the energy regime between $\Lambda_{1}$ and $\Lambda_{2}$, new interactions between the old particles might become important. $T_{2}$ is then constructed by including these new interactions in the Lagrangian density of 
$T_{1}$. In order to save the phenomena accounted for already by $T_{1}$, some of the parameters of $T_{1}$ might have to be changed and new parameters have to be adjusted appropriately.

2. There are new particles between $\Lambda_{1}$ and $\Lambda_{2}$.

This case is, of course, the more complicated one. $T_{2}$ is now constructed in several steps. First, the masses and charges of $T_{1}$ have to be adapted to the new energy scale; again, this is done by solving the renormalisation group equations. Second, all new particles which show up in the energy regime between $\Lambda_{1}$ and $\Lambda_{2}$ have to be identified. Are they fermions or bosons? What is their mass and charge (on the scale $\Lambda_{2}$ )? How do they couple to the other particles? The formalism of quantum field theory presents a tool box to systematically construct the new terms in the Lagrangian density of $T_{2}$. In many cases, the relevant coupling constants have to be adjusted to experimental results. This procedure therefore has a theoretical (or a priori, if you like) and an experimental (or a posteriori) component. The structure of the new terms follows from the general formalism of quantum field theory. The masses, charges and coupling constants have to be determined on the basis of experiments. This procedure is, of course, not completely theory-free. The determination of the relevant parameters takes place on a given energy scale, and auxilliary (or measurement) theories have to be used to determine their numerical values. These measurement theories also work on a given energy scale, and consistency must be achieved in this whole process.

There are several examples for the bottom-up approach in physics. The Fermi theory of weak interactions, discussed in Section 2.1.2, is a good example of a theory which had to start from scratch. Doing so, one might be mistaken, for example, when it comes to specifying the correct interaction between the relevant particles, as the discovery of parity violation for weak interactions shows. The work based on taking the general theory of relativity as an EFT is a good example of case 1 mentioned above. Here new interactions are included which correct Einstein's theory at higher energies. The new theories obtained by following this approach have been interpreted tentatively as low-energy limits of a quantum theory of gravity. We do not know this theory yet, but following the EFT approach might eventually lead to new ideas as to what such a theory might look like (Donoghue, 1994a,b). The problem here is of course that there are almost no experimental data available which can be used to fix the parameters in the new theory (as in the case of Fermi's theory). It might, however, be possible to derive these parameters from candidate theories for a quantum 
theory of gravity. Supersymmetric extensions of the Standard Model are an example of case 2. Here the Standard Model is essentially duplicated by including the supersymmetric partners of all particles which are already present in the Standard Model (Meissner, 1992). Again, the problem here is that there are no experimental data yet that can be used to fix the new parameters (such as the masses of the supersymmetric partners of the leptons and quarks).

\subsubsection{The top-down approach}

This strategy starts with a more fundamental theory which is vaild on a given energy scale $\Lambda_{1}$. The aim is now to construct an EFT for lower energies $\Lambda_{2}<\Lambda_{1}$. There is a systematic procedure for getting these low-energy theories. Once the original theory is renormalisable and the decoupling theorem holds, a tower of EFTs can be uncovered in this way. A typical example of this strategy is the theory of Euler and Heisenberg, discussed in Section 2.1.1. Here, a purely photonic theory was obtained from QED by eliminating all electronic degrees of freedom. The resulting EFT is then valid for photons whose energy is much smaller than the rest mass of the electron. Other examples of this strategy are the various attempts to justify some kind of superstring theory. These theories cannot be tested experimentally at a typical energy of a superstring. Instead, systematic low energy expansions are carried out in order to obtain low-energy footprints of the high energy regime of this theory. Yet another example of the application of the top-down strategy is provided by nuclear and particle physics. I will look at this case in some more detail in the next section.

\section{Theorising in Nuclear and Particle Physics: A Case Study}

The present situation in theoretical nuclear and particle physics is rather involved. On the one hand, there is the Standard Model, a renormalisable and well-confirmed theory which should, in principle, account for all phenomena which are not gravitational. On the other hand, there are all sorts of models and EFTs which are often used in practical applications. Especially in the sector of the Standard Model which deals with strong interactions, a plurality of theoretical accounts can be identified. They all seem to coexist peacefully, and they all seem to complement each other in a way which I will investigate in more detail below. In this section, I shall focus on the physics of the strong interaction only and first introduce quantum chromodynamics, the underlying theory, as well as some typical models and EFTs in this part of physics 
(Section 3.1). By focusing on their respective functions in the research process, Section 3.2 argues that all of them are indispensable and Section 3.3 points out various interrelations between them. This case study will be the basis of my argument for some variant of pluralism in the next section.

\subsection{Theories, models and EFTs}

For a long time, the physics of strong interactions lacked a fundamental theory. There were phenomenological models, all of which could be applied for some purposes, but all of which had their well-known limits. Among the models used extensively in nuclear physics are the liquid drop model and the nuclear shell model. The liquid drop model helps to understand nuclear fission, as Niels Bohr and John A. Wheeler pointed out, but fails to explain why certain configurations of protons and neutrons are particularly stable. These 'magic numbers' of protons and neutrons can be naturally explained with the nuclear shell model which has, in turn, other deficiencies. Among the models of the constituents of the nuclei (protons, neutrons and pions) are various bag models, chiral quark models, the purely bosonic Skyrme model, and approaches which utilise sum rules derived in the spirit of the $S$-matrix tradition.

All these models were considered provisional at best, and applied and studied because of a lack of a more satisfactory alternative. Fortunately, this alternative was found in the early nineteen-seventies. ${ }^{15}$ Quantum chromodynamics (QCD) was born, but it soon turned out that tractable applications of this theory could only be obtained in the high-energy regime. Nuclear physics and the theory of hadron structure remained almost completely unaffected and developed quite independently for a long time. There have been some attempts to derive low-energy results from QCD, but these endeavours turned out to be technically extremely hard and rather uninteresting and unilluminating. Other QCD-inspired research in hadron physics involved the qualitative modelling of features of QCD such as confinement and dynamical chiral symmetry breaking. These models (see Section 3.1.2) have been quite successful, but their formal relation to QCD is far from clear. This is where EFTs come in handy. EFTs allow a systematic low-energy expansion of QCD, and many of the old models could be given a more solid foundation.

\subsubsection{The theory: quantum chromodynamics}

QCD is generally considered to be the fundamental theory of strong interactions.

\footnotetext{
${ }^{15}$ For a reconstruction of this development and for an analysis of the role models played in this context see Hartmann (1995a,b).
} 
It is a renormalisable gauge theory, and its fundamental entities are the fermionic quarks (spin-1/2) and the bosonic gluons (spin-1). There are six different kinds of quarks ('flavours'): up, down, strange, charm, bottom and the recently discovered top. Besides spin and flavour, quarks have an additional degree of freedom which is called 'colour'. Gluons, the exchange particles of the strong interactions, show up in eight different kinds and, unlike photons in QED, directly interact with each other. This fact, which is a consequence of the internal colour structure of the gluons, along with the large value for the coupling constant of QCD, makes actual calculations very complicated and involved. The self-interaction of the gluons follows mathematically from the non-commutativity of the generators of the corresponding gauge group, colour-SU(3). Here is the Lagrangian density of QCD:

$$
\mathcal{L}_{Q C D}=\bar{\psi}\left(\mathrm{i} \gamma_{\mu} D^{\mu}-\hat{m}_{0}\right) \psi-\frac{1}{4} F_{k \mu \nu} F_{k}^{\mu \nu}
$$

$\psi$ represents the quark field and $F_{k}^{\mu \nu}$ (with $k=1, \ldots, 8$ for the eight gauge degrees of freedom) is the field strength tensor associated with the gluons. The operator $D^{\mu}$ fixes the gauge invariant coupling of the quarks and the gluons and $\hat{m}_{0}$ is the mass matrix of the quarks; the quarks which show up in the Lagrangian density of QCD are also called 'current quarks', as opposed to the much heavier 'constituent quarks' of non-relativistic quark models. This matrix cannot be deduced from first principles and has to be adjusted to experimental data.

For most low-energy applications, exact consequences of Eqn (19) can only be obtained numerically with a method called lattice gauge theory (see Montvay and Münster, 1994). Here quark and gluon fields are defined on a lattice with a finite spacing $a$; exact results can be obtained by running extensive computer simulations with finite $a$ and extrapolating the results to the continuum limit $a \rightarrow 0$. Although this method also suffers from technical problems, my main point about lattice gauge theory is that it effectively works like a black box. Technical problems aside, lattice gauge theory produces the exact results of QCD and hence makes tests of this theory possible. However, it does not reveal more about the concrete mechanisms which account for the calculated result. Like a black-box theory, lattice gauge theory yields consequences of a theory, but it does not produce insight and understanding. ${ }^{16}$

This account of 'black-boxism' does not square with traditional black-box theories such as behaviourism. These theories were criticised for not providing a detailed mechanism for the dynamics of a system. According to Bunge (1964), these

\footnotetext{
${ }^{16}$ This point is elaborated in Hartmann (1999).
} 
mechanisms have to be provided by a fundamental theory. ${ }^{17}$ In the case of strong interactions, QCD does indeed specify the overall dynamics of the system; there are quarks and gluons, and these entities interact in a very complicated way with each other according to the Lagrangian density of QCD. But not much more can be said: the rest has to be done numerically with the help of high-powered computers (cf. Lepage (1994)). And computers function like a black box. All possible Feynman diagrams are summarised, although, perhaps, only a few of them (or a certain subclass of them) produce almost the whole effect under investigation. A knowledge of these actually relevant processes would produce insight and understanding. Lattice gauge theory does not produce this insight, and QCD is, therefore, effectively a black-box theory.

In order to learn something about the actually relevant processes, models and EFTs are applied. While EFTs can be directly obtained from QCD by following well-defined procedures, models usually extract one or more of the general features and consequences of the theory and explore their implications. Some of these general features and consequences are well known. Among them are the following three (Donoghue et al., 1992).

First, QCD is asymptotically free. This means that quarks move freely at very high energies. At low energies (energies of the order of the rest mass of the proton), the reverse effect shows up and quarks and gluons interact very strongly with each other. This is why perturbation theory, which works so well in QED, cannot be applied here.

Second, QCD exhibits quark confinement. First introduced to account for the fact that no one ever observed a free quark, it now seems clear that quark confinement is a strict consequence of QCD. But what does confinement really amount to? This is not so clear and there are several options to be found in the literature. Some argue that the interaction between the quarks increases with their distance, others favor a model according to which quarks are bound inside some solid sphere which prevents the existence of free quarks. And there are other, more technical proposals (for details see Hartmann (1999)).

Third, low energy QCD is (almost) chirally invariant and exhibits dynamical chiral symmetry breaking. Unlike confinement, this phenomenon is well understood. Here is the basic idea. The masses of the quarks in the Lagrangian density of QCD (see Eqn (19)) are very small (about $10 \mathrm{MeV}$ ) compared to the typical energy scale of strong interactions (about $1 \mathrm{GeV}$ ). Let us therefore assume that quarks are massless. The

\footnotetext{
${ }^{17}$ For an interesting discussion of the role of phenomenological theories in physics see also Heisenberg (1966).
} 
Lagrangian density of QCD then exhibits another symmetry, called chiral symmetry. As a consequence of this symmetry, so-called left-handed and right-handed eigenstates of the QCD Hamiltonian cannot be distinguished energetically. Every hadron which is an eigenstate of the QCD Hamiltonian should have a chiral partner with the same mass, but with opposite chirality. Now, these chiral partners do not seem to exist. There is, for example, no other particle with the mass and charge of the proton, but with opposite chirality. ${ }^{18} \mathrm{~A}$ way out of this difficulty is to assume that chiral symmetry is dynamically broken. This means that the interaction itself breaks the symmetry so that a large mass gap between the chiral partners emerges. The pion emerges as the corresponding Goldstone boson of the broken symmetry. It should be noted, however, that chiral symmetry is also explicitly broken due to the nonvanishing of the values of the current quark masses in the Lagrangian density of QCD. This effect has some interesting consequences, such as the finite pion mass.

\subsubsection{Models}

I take a model to be a set of assumptions (added, perhaps, by diagrams, sketches, and other visualisations), where some of these assumptions might be inspired by a theory. All other assumptions specify the concrete object or system under consideration. Phenomenological models, like the ones in hadron physics, use theories like a tool box; they pick some of the relevant features of (at least) one theory, fit these into a larger theoretical framework (which might be different from the one employed by the theory), and explore the consequences of the assumptions made. This procedure allows for models to be used as probes for the features of the underlying theory (Hartmann, 1999). The deductive relation between a model and an underlying theory is, however, not at all clear.

Among the relevant features of QCD at low energies are quark confinement and the dynamical breaking of chiral symmetry. While bag models concentrate on the first feature, chiral quark models explore the consequences of QCD's second main feature. The first and conceptually easiest bag model is the MIT-Bag Model (see Mosel, 1999, Ch. 16). Here quark confinement is included in the model assumptions by restricting the motion of quarks to a finite region in space, the 'bag'. Mathematically this is done by imposing an appropriate boundary condition to the quark wavefunctions which are assumed to be a solution of the (free) Dirac equation for relativistic particles. Bag models like the MIT-Bag Model therefore do not operate in the framework of quantum field theory (such as QCD), but in the framework of relativistic quantum mechanics which is mathematically easier to handle.

\footnotetext{
${ }^{18}$ The chirality is given by the projection of the spin on the momentum of the particle.
} 
Chiral models explore the consequences of chiral symmetry and its dynamical breaking. Some of these models take only quark degrees of freedom into account (such as the Nambu-Jona-Lasinio model), others (such as the Skyrme model) neglect quark degrees of freedom completely and describe hadrons in terms of scalar and pseudoscalar meson fields, while yet other models favour an hybrid account of quark and meson degrees of freedom (such as the soliton models of the Friedberg-Lee type) (see Mosel, 1999, Ch. 17). These models have a long history which started in the days of the $S$-matrix programme and the work on current algebra in the nineteen-fifties and sixties. In this context, plenty of experimentally well-confirmed relations between hadron masses have been derived from the assumption of chiral symmetry only. A famous example is the Gell-Mann-Oakes-Renner relation which relates properties of the pion (its mass $m_{\pi}$ and its decay constant $f_{\pi}$ ) to quark properties:

$$
m_{\pi}^{2} f_{\pi}^{2}=-\frac{m_{u}+m_{d}}{2}<\bar{q} q>
$$

where $<\bar{q} q>\approx(-250 \mathrm{MeV})^{3}$ is the so-called quark condensate.

\subsubsection{Effective field theories}

The most popular EFT based on QCD is Chiral Perturbation Theory, developed by Steven Weinberg, Heinrich Leutwyler and others in the nineteen-eighties. I will give a short outline of this approach along the lines of Leutwyler (1994).

The main idea of Chiral Perturbation Theory is to expand the Lagrangian density of QCD in terms of a typical momentum for the process under consideration. For the sake of simplicity let us assume for a moment that the current quark masses vanish $\left(m_{u}=m_{d}=0\right)$ and that there are only two quark flavours. This last assumption is reasonable in the low-energy regime of about $1 \mathrm{GeV}$.

In order to get the desired momentum expansion, one first replaces the quark and gluon fields of QCD by a set of pion fields which are, as pointed out above, the Goldstone bosons of the theory due to the dynamical breaking of chiral symmetry. These fields can be conveniently represented by a $2 \times 2$ matrix $U(x) \in \mathrm{SU}(2)$. Next, the Lagrangian density of QCD is expressed exclusively in terms of $U(x)$. After this decisive step in the derivation, quark and gluon degrees of freedom do not show up in the Lagrangian density anymore. One obtains:

$$
\mathcal{L}_{Q C D}=\mathcal{L}_{e f f}\left(U, \partial U, \partial^{2} U, \ldots\right) .
$$

It turns out that a low energy expansion of this Lagrangian density can be obtained by expanding $\mathcal{L}_{Q C D}$ in terms of the derivatives of $U(x) .{ }^{19}$ The Lorentz invariance

\footnotetext{
${ }^{19}$ This follows from the observation that the momentum operator is given by $p_{\mu}=-\mathrm{i} \partial_{\mu}$.
} 
of the whole Lagrangian density implies that only terms with an even number of derivatives show up in the truncated expression. One obtains:

$$
\mathcal{L}=\mathcal{L}_{\text {eff }}^{2}+\mathcal{L}_{\text {eff }}^{4}+\mathcal{L}_{\text {eff }}^{6}+\ldots
$$

The requirement of chiral symmetry very much constrains the form of the terms in this expansion. The second-order contribution is given by:

$$
\mathcal{L}_{\text {eff }}^{2}=\frac{1}{4} F_{\pi}^{2} \operatorname{tr}\left[\partial_{\mu} U^{\dagger} \partial^{\mu} U\right]
$$

This term is essentially determined by the decay constant of the pion $\left(F_{\pi}\right)$. The next term of order $p^{4}$ is already a bit more complicated:

$$
\mathcal{L}_{\text {eff }}^{4}=\frac{1}{4} l_{1}\left(\operatorname{tr}\left[\partial_{\mu} U^{\dagger} \partial^{\mu} U\right]\right)^{2}+\frac{1}{4} l_{2} \operatorname{tr}\left[\partial_{\mu} U^{\dagger} \partial_{\nu} U\right] \operatorname{tr}\left[\partial^{\mu} U^{\dagger} \partial^{\nu} U\right] .
$$

It turns out that these first two terms suffice already for many practical applications.

Why is this procedure called 'Chiral Perturbation Theory'? The reason is this: we have, so far, assumed that the quark masses vanish and that chiral symmetry is hence an exact symmetry of QCD. This is an approximation since chiral symmetry is explicitly broken due to the finite (though small) current masses of the quarks. These effects are taken into account in another perturbation expansion in the quark masses.

The whole procedure of Chiral Perturbation Theory therefore consists of two power series expansions, one in some typical momentum, and the other in the mass matrix $m=\operatorname{diag}\left(m_{u}, m_{d}\right)$ of the quarks. In order to be consistent, one contribution of a quark mass term in the expansion must correspond to two powers of the momentum. In leading (i.e. second) order one obtains:

$$
\mathcal{L}_{\text {eff }}^{2}=\frac{1}{4} F_{\pi}^{2} \operatorname{tr}\left[\partial_{\mu} U^{\dagger} \partial^{\mu} U\right]+\frac{1}{2} F_{\pi}^{2} \operatorname{tr}\left[m\left(U+U^{\dagger}\right)\right] .
$$

It is interesting to note that many of the phenomenological models and current algebra relations (low-energy theorems) derived in the nineteen-sixties and seventies can be strictly deduced from QCD and summarised in a compact fashion (cf. Ecker, 1995).

While the original programme of Chiral Perturbation Theory only aimed at applications in hadron physics, the more complicated task of deriving EFTs for nuclear physics from QCD has also been undertaken. This remarkably popular and successful research programme is reviewed by Van Kolck (1999). It demonstrates how the relation between theories of several domains (nuclei-protons, neutrons, and pions - and 
quarks) can be studied in a mathematically controlled way. This fact has implications for the reductionism debate, which will be discussed in Section 4.2.

\subsection{Functions of theories, models and EFTs}

Theories, models, and EFTs have various functions in actual scientific practice. They are more or less efficient tools in the process of theorising, helping scientists to reach certain cognitive goals. It turns out that none of these tools serves all the functions scientists are interested in. Consequently, a suitable combination of them has to be applied. We will come back to this in Section 4.1.

\subsubsection{The functions of theories}

Theories have a wide scope of applicability. QCD, for example, should apply to all phenomena governed by strong interactions. It should not only account for the properties of protons and neutrons, but also for whole nuclei and their interactions as well as for astrophysical objects such as neutron stars. Theories provide a coherent account of a large class of phenomena: they unify phenomena which, at first sight, do not have much to do with each other. When it comes to calculations, theories (such as QCD) give the most precise values for the quantities in question. They are therefore also good tools to predict new effects. Theories constrain the assumptions made in models, and may also suggest models (such as the hadron models mentioned above).

However, the price for universal scope and predictive accuracy is that the theory does not provide local understanding of the relevant physical processes. Usually, the theory can only be solved numerically and the entities employed by it, such as quarks and gluons, are 'too far away' from the phenomena in question. An understanding of why neutron stars eventually collapse, for example, is hardly achieved by referring to the dynamics of the myriads of quarks and gluons which supposedly constitute these astrophysical objects. Theories produce global understanding by fitting an object or system under consideration into a bigger framework, but tend to fall short in their efforts to produce local understanding (see also Section 4.3).

\subsubsection{The functions of models}

Models, on the other hand, produce local understanding. They often go with a causalmechanistic story and aim at capturing the essential physics of a phenomenon in a few assumptions (with only a few parameters), just as a caricature represents a person with a few brush lines. As a consequence of this, models are easy to handle mathematically (compared to a more fundamental theory), and deductive consequences of 
the model can be obtained in an efficient manner. This pragmatic (or computational) superiority of models to theories can hardly be over-estimated (see Humphreys, 1994, 1995). Models are also heuristically very important; they often play a decisive role in the construction of more fundamental theories (such as QCD, as I showed in detail in Hartmann (1995a,b)) or suggest strategies to derive EFTs from a more fundamental theory. ${ }^{20}$

Among the drawbacks of models are the following. The assumptions made by models often lack a deeper foundation; sometimes they are just ad hoc in order to save a phenomenon. As the case study has shown, there might not be a 'controlled' deductive relation between the model and an underlying theory, and if a derivation of the model from the theory is actually carried through, further assumptions have to be made to obtain the model, and these assumptions (which might turn out to be more dubious than the assumptions made by the original model) again require a justification, and so on, ad infinitum. The parameters which enter a model are sometimes derived from the theory; often, however, they are simply adjusted to experimental results. So they often require a deeper theoretical underpinning. Assumptions made by models might contradict assumptions made by other models, or by a theory for that matter. The MIT-Bag Model, for example, violates chiral symmetry, and many chiral quark models lack confinement. And yet, as I have argued in Hartmann (1999), the models and the underlying theory are bound together in some sense. There often is a story which connects the vocabulary of the model to the vocabulary of the underlying theory even if there are no formalised ontological bridges in the sense of Rohrlich (2000). We shall come back to this in Section 4.1.

\subsubsection{The functions of EFTs}

EFTs share many of the functions of theories and models. Like models, they provide a local, intuitive account of a given phenomenon in terms of the degrees of freedom which are relevant at the energy scale under consideration. They are relatively easy to solve and to apply, and they are heuristically useful. This is demonstrated by the Fermi theory and the $V-A$ theory which eventually led to the Standard Model, as well as by the EFTs which are used to test the low-energy regime of a future quantum theory of gravity. Like theories, EFTs are part of a bigger picture or framework, from which they can be derived in a controlled way. They help to make predictions and to test the theory they relate to. EFTs avoid the disadvantage of theories of being 'too far away' from the phenomena.

In practice, however, EFTs often contain more adjustable parameters than a

\footnotetext{
${ }^{20} \mathrm{~A}$ more complete list of the various functions of models can be found in Hartmann (1999).
} 
model of the same system. Besides, EFTs are only applicable if the energy scales of a system separate well. That is why EFTs work well in particle physics, but do not work so well in the physics of complex systems. Here models and perhaps more fundamental theories are required. It should also be noted that EFTs are closely related to the general framework of QFT. If this framework theory breaks down at some energy and, say, a superstring theory takes over, the whole idea of EFTs might also be vitiated. Then it would have to be clarified in which sense the old EFTs can be recovered or obtained within a certain limit from the new theory.

\subsection{Relations between theories, models and EFTs}

The theories, models, and EFTs discussed in this section are intimately related to each other. There are various interactions and dependencies as well as conceptual and cognitive relations between these tools which I will now point out by going through the material presented in the case study above.

Theories and models are often not related deductively. However, theories may inspire models which pick out a feature of a more fundamental theory, such as confinement or chiral symmetry in the case of QCD, and embed it in a less complex theoretical framework (such as non-relativistic quantum mechanics). By doing so, models function as a probe to explore the consequences of just one aspect of the theory. This leads to a better understanding of the physics represented by the theory. Theories may also be used to fix the numerical value of the parameters of a model. Many quark models, for example, employ the strong coupling constant which is taken from QCD. Other models have adjustable parameters which can be calculated directely from the underlying theory (although this is often not done in order to have more freedom to adjust the model to experimental data). Models, on the other hand, often play a role in the construction process of a theory (as the QCD-example demonstrates).

Models and EFTs are not always easy to distinguish. Sometimes, a newly developed EFT or some consequence of it turns out to be identical to a model developed many years earlier. An example of this is the work on current algebra. Many of the results which were derived in this framework since the nineteen-sixties turn out to be consequences of chiral perturbation theory. The EFT then provides additional support for the model. Some EFTs are treated like models because no attempt has been made to calculate, for example, the coupling constants and renormalised masses from first principles. They are simply fitted to experimental data. In some cases, models are employed to obtain these parameters.

EFTs and theories may be related in a deductive sense, provided that there is 
a theory. EFTs then serve to apply and test the theory because they are easier to handle mathematically. They point out the relevant mechanisms at a given energy scale, which helps to better understand the physics covered by the theory. If there is no theory, following the bottom-up strategy of constructing a tower of EFTs might eventually give scientists a hint as to where to look for a more fundamental theory. But of course, there is no direct way from, say, QED to quarks; just using the tricks of the renormalisation group will not take you from here to there. This is where creativity and imagination comes in.

Most important are the cases where theories, models and EFTs complement each other. The establishment of dynamical chiral symmetry breaking as a feature of QCD, for example, resulted from the interaction of all three approaches. Lattice gauge calculations suggested models, consequences of models were used to derive an EFT, which in turn inspired other models and allowed for analytical results. It is this interaction between various tools that makes scientific research so exciting.

\section{Some Philosophical Lessons}

I will now draw some more general conclusions from this case study. The main point I would like to make is that theories, models, and EFTs are indispensible tools in scientific research. They complement each other in a way which will be analysed in some more detail in Section 4.1. Sections 4.2 and 4.3 focus on the consequences for the reductionism debate and the controversy about scientific explanation.

\subsection{Pluralism and coherence}

Generalising the results of the case study, the following picture of theorising in physics emerges. Scientists use a variety of theoretical tools; among these tools are theories, models and EFTs. All of them have specific functions, and all of these functions are required.

This has consequences for the notorious debate about the final theory. Setting aside worries that we will anyway never be in the position to write down this theory, a final theory faces at least three problems. First, it will be 'too far away' from the phenomena we experience directly or in a laboratory. A final theory is therefore unlikely to provide a local understanding of these phenomena. Models and EFTs are still needed for this. Second, the final theory cannot be applied without various additional (model-) assumptions about the concrete system under investigation. Besides, low-energy expansions have to be carried out in a systematic way. This is 
where EFTs will come in handy. And third, we will probably never arrive at a final theory without a supporting scaffolding of various models and EFTs. This has been the case for all theories of physics so far. So there is no reason to believe that things will be different for a final theory.

Let us now consider whether models are likely to dominate theoretical science as some philosophers of science think (see Cartwright, 1999). In our case study, none of the models we looked at was taken seriously by scientists if there was not at least a qualitative story which connected the model to a more fundamental theory. And even before the formulation of QCD, physicists did not consider the plurality of nuclear and hadron models to be a satisfactory state of affairs. Theories are needed to inspire the development of models and to present a framework for the various models.

EFTs are also not likely to make the the other two tools obsolete. Although EFTs share many of their functions with theories and models, they will not be able to fulfil all of these functions. To illustrate this point, let us distinguish two cases. First, there is a fundamental theory from which specific EFTs can be deduced. Then, of course, the EFT depends on the fundamental theory which is then still an essential part of our theorical account of the world. Second, there is no fundamental theory. In this case the 'recipe' which is part of the bottom-up EFT ideology is to try and construct a tower of EFTs. More and more new particles will be added to the theory, all of them being 'elementary' in a certain sense, and all of them might couple to all the other particles known so far. But the resulting theory will not be of much value; it is simply too complicated, and its predictive power will go down just as the predictive power of the Ptolemaic system went down when more and more epicycles were added (see Forster, 2000). Instead, theorists will search for a more fundamental theory which will reduce the contingency that goes along with the tower-construction strategy.

The upshot of all this is that theories, models and EFTs are each indispensable tools of theoretical research in physics. But how are they related? Obviously there are logical contradictions between various models, and also between QCD and EFTs. Nevertheless, they all hang together in some sense. Sometimes there is an approximate deductive relation between, for example, QCD with an EFT at a given energy scale. In the framework of Chiral Perturbation Theory, both are related through an ontological bridge (Rohrlich, 2000), i.e. the identification of the terms involving quark degrees of freedom in the Lagrangian density of QCD with the bosonic field $U(x)$. But the connections are not always so tight. Sometimes there is only a plausible story which relates the vocabularies of a model and QCD and which sets the model in the bigger framework provided by a theory. This story can be interpreted as a semantic bridge between the model and the theory. There is a whole continuum 
of relations between theories, models and EFTs which range from strict reductive relations through ontological bridges to rather vague associations through semantical bridges. It is these bridges which integrate all these approaches in a coherent whole (note that coherence comes in degrees!). It is tempting to reconstruct this picture in terms of the probabilistic model of the coherence of a belief set suggested in Bovens and Hartmann (2000) and developed in Bovens and Hartmann (forthcoming). The various bridges between a model $M$ and a theory $T$, for example, could then be modelled by the conditional probability $P(M \mid T)^{21}$. While this discussion refers to the statics of scientific theorising (how do the theories and models at a given time hang together?), a discussion of the role of considerations concerning coherence in the dynamics of successive scientific theories can be found in Hartmann (forthcoming).

\subsection{Reduction and emergence}

The issue of reduction is probably the one which so far has got most attention in the philosophical literature about EFTs. Much of this debate relates to the radical conclusions Cao and Schweber draw in their article from 1993. The authors claim to have 'found that the recent developments support a pluralism in theoretical ontology, an antifoundationalism in epistemology and an antireductionism in methodology' ( $\mathrm{p}$. 69 ). I will evaluate these claims on the basis of the case study presented in the last section.

First, ontological pluralism. This thesis is based on the observation that empirical reality seems to be organised in a multitude (infinity?) of quasi-autonomous layers. Each layer has its own ontology, and this ontology is to a considerable extent independent of the physics at higher energies. Only when the probing energy approaches the cut-off of a given layer, do effects of the higher layers turn out to have some influence. This influence might lead to a renormalisation of the mass and charge parameters, but it might also lead to the insight that the ontology used so far is not really fundamental. Nuclei turned out to be composed of nucleons and pions, and nucleons and pions turned out to be composed of quarks and gluons. Cao and Schweber's claim of ontological pluralism rests on the assumptions, as Robinson (1992) has pointed out, 'that we can build up our ontological commitments in QFT only by the method of first identifying the referring terms of the theory we accept. But to accept this form of realism - theoretical realism - is to deny that we can build up our ontological commitments through experiment in the absence of theory' (p.

\footnotetext{
${ }^{21}$ Given the prior probabilities for $M$ and $T, P(M)$ and $P(T), P(T \mid M)$ can be obtained by Bayes Theorem.
} 
403). I would not go as far as Robinson and subscribe to some variant of entity realism. Rather, I would point to the role which other theories and their interrelations play when it comes to establishing the ontology of the world. And given these other theories and their interrelations it seems clear that ontological pluralism can at best be defended as a pragmatic thesis. ${ }^{22}$ We are not trapped in the language game of one theory. Given these other theories it might be interesting to take EFTs as a case study in emergence. How do the properties of nucleons, for example, emerge from the complicated interplay of quarks and gluons? This is a physics problem, and the role of symmetry breaking mechanisms has to be addressed in detail. It should also be noted that Cao and Schweber's talk of quasi-autonomous domains rests on the validity of the decoupling theorem. As I have explained in Section 2.2.3, this theorem can only be proven if there is a underlying renormalisable theory and if the energy scales of the particles separate. While the second assumption might not be fulfilled empirically, the first assumption renders many of Cao and Schweber's more radical conclusions implausible since they are based on the assumption that there is no underlying theory.

Second, epistemological antifoundationalism. According to this thesis, quasiautonomous layers do not only have their own ontology, but also have their own 'fundamental' theory. Since none of the layers is distinguished, none of the theories is the fundamental one. There is no foundation for all other theories, and there is no (and there will never be a) final theory which entails all other theories. Cao and Schweber know that this is a metaphysical thesis, but they think that it is well supported by the practice of science. And indeed, a final theory seems to be as far away as it was twenty years ago. But this, of course, does not imply that there will never be a final theory. Leaving metaphysical questions aside, it seems to be philosophically more interesting to examine the formal relations between the theories, models and EFTs we have already. As I argued in Section 4.1, the relation between some underlying theory and an EFT can be reconstructed along the lines of Rohrlich's (2000) two-step model: first, a deductive relation between the two formalisms has to be obtained. This typically involves approximations and a limiting process. Second, ontological bridges between the incommensurable concepts of both theories have to be established. This model, designed to account for the relation between mature theories, does not help to analyse the relation between a model and a theory, which is typically much more involved. This calls for detailed case studies.

Third, methodological antireductionism. This position advocates the bottom-up EFT research strategy which is also favoured by many pragmatically-minded physi-

\footnotetext{
${ }^{22}$ For another account of ontological pluralism see Rohrlich (1988).
} 
cists. Howard Georgi, for example, writes:

The philosophical question underlying old-fashioned renormalizability is this: How does this process end?

It is possible, I suppose, that at some very large energy scale, all nonrenormalizable interactions disappear, and the theory is simply renormalizable in the old sense. This seems unlikely, given the difficulty with gravity.

It is possible that the rules change dramatically, as in string theory.

It may even be possible that there is no end, simply more and more scales as one goes to higher and higher energy.

Who knows?

Who cares?

In addition to being a great convenience, effective field theory allows us to ask all the really scientific questions that we want to ask without committing ourselves to a picture of what happens at arbitrarily high energy (Georgi, 1993, p. 215).

Georgi recommends the bottom-up EFT strategy for pragmatic reasons. Unlike a final theory, EFTs can be systematically tested experimentally, and this is taken to be a feature any acceptable scientific theory should have:

My personal suspicion is that Nature is much more imaginative than we are. If we theorists approach her study with the proper respect, if we recognize that we are parasites who must live on the hard work of our experimental friends, then our field will remain healthy and prosper. But if we allow ourselves to be beguiled by the siren call of the 'ultimate' unification at distances so small that our experimental friends cannot help us, then we are in trouble, because we will lose that crucial process of pruning of irrelevant ideas which distinguishes physics from so many other less interesting human activities (Georgi, 1989, p. 457).

Georgi does not commit himself to a view concerning the possible existence of a final theory. Whether it exists or not is not a question which can be settled in the laboratory. Maybe the tower of EFTs never ends. It would, however, be a mistake to stop taking the possibility of a final theory into account. Michael Redhead also argues this point:

[F]rom a point of view of methodology of science a recurring theme has been the search for an ultimate underlying order characterized by simplicity and symmetry that lies behind and explains the confusing complexity 
of the phenomenal world. To subscribe to the new EFT programme is to give up on this endeavour and retreat to a position that is admittedly more cautious and pragmatic and closer to experimental practice, but is somehow less intellectually exciting. Perhaps one should not allow such considerations to enter into one's evaluation of a scientific programme, but to my own taste, the regulative ideal of an ultimate theory of everything remains a powerful aesthetic ingredient motivating the existence of the greatest intellectual ingenuity in the pursuit of what may admittedly, in itself, be an illusory goal. But that after all is the proper function of regulative ideals (Redhead, 1999, p. 40)

\subsection{Explanation and understanding}

One of the major aims of science is to explain phenomena. Although the concept of explanation is pretty vague, an acceptable explanation should show (1) how the phenomenon under consideration reached its present state and (2) how it fits into a larger theoretical framework. Although these two requirements do not exclude each other, it remains to be seen if both can be fulfilled by the same scientific theory or model. This is not clear to start with, and philosophical theories of explanation therefore usually concentrate on one of these requirements - a task which turns out to be hard enough as the controversial nature of the debate over the last four decades or so impressively shows (Salmon, 1989).

According to the causal/mechanical account, pioneered by Salmon and others (see Salmon, 1998), a phenomenon is explained by providing a mechanism which produces the effect under consideration. This mechanism is often given by a model (or an EFT for that matter), as the MIT-Bag Model illustrates. Here quarks are confined to a hard sphere in which they can move freely apart from occasional bounces off the inner side of the bag, a situation which can be easily visualised classically (see Section 3.1.2). The mass of the proton, for example, can then be determined by summing up the kinetic energies of the quarks and the potential energy of the bag. Explanations of this kind produce local understanding, but lack global understanding because no general principles are required to specify the mechanism.

According to the unification account, developed by Friedman and elaborated by Kitcher (1989), a successful explanation fits the explanandum in a general framework. This view, which is a distant descendant of the original Hempel-Oppenheim account, supports the intuition that something is explained if it is integrated in a larger theoretical context. Explanations of this kind are provided by theories such as QCD. An explanation of the mass of the proton, for example, goes like this: there are quarks and gluons coupled to a state with the quantum numbers of the proton 
and interacting in a very complicated way. Deductions from the Lagrangian density of QCD, facilitated by high-powered computers, then yield the result of $938 \mathrm{MeV}$. As I have argued in Section 3.1.1, this account does not produce local understanding. By integrating the proton in a bigger framework it produces, however, global understanding.

I think that the question which of the two accounts of explanation is the right one is misguided. The case study presented in Section 3 has shown that both accounts complement and interact with each other. The account of scientific explanation I consider to be in accordance with scientific practice is therefore a pluralist one: science studies a given phenomenon from various theoretical perspectives, all of which reveal some explanatory information about the phenomenon in question. Putting all of them together should result in a coherent explanatory account of the phenomenon. It should be noted that Salmon (1998, pp. 73f) also acknowledges a rapprochement of the causal/mechanical account and the unification account of scientific explanation in his recent book.

\section{Conclusions}

Science is a complex and involved activity. All simple reconstructions of it will probably fail. Generalisations based on the work on theory unification in particle physics, for example, are as hasty as the philosophical conclusions some now draw from the current interest in EFTs among physicists. Science usually does not address issues such as unity, reductionism, and what the characteristics of a good explanation are. Rather, scientists use a plurality of interrelated conceptual tools, and explanations are obtained by attacking a phenomenon from a variety of theoretical perspectives. It is this pluralism of tools which is good for science and which makes science flourish.

Acknowledgements - This article has a long history. Prior versions of it were presented at the Centre for Philosophy of Natural and Social Sciences at the London School of Economics in 1994, the '2nd Congress of the German Society for Analytical Philosophy' in Leipzig, Germany (1994), and at the 'Congress for Logic, Methodology and Philosophy of Science' in Florence, Italy (1995). Thanks to the audiences, especially Jeremy Butterfield, Nancy Cartwright, Margaret Morrison, Michael Redhead, and Manfred Stöckler for many suggestions and stimulating discussions. Luc Bovens, Alfred Nordmann, Fritz Rohrlich, and the editors commented on a draft of this article and suggested various stylistic and substantial improvements. Thanks also to Jim Lennox and the Center for Philosophy of Science at the University of Pittsburgh 
in whose stimulating atmosphere this article was completed, and the Alexander von Humboldt Foundation (Bonn, Germany) for financial support.

\section{References}

Appelquist, T. and Carazzone, J. (1975) 'Infrared Singularities and Massive Fields', Physical Review D11, 2856-2861.

Becker, W., McIver, J. and Schlicher, R. (1989) 'Testing the Photon-Photon-Sector of Quantum Electrodynamics With Free-Electron Lasers', Journal of the Optical Society of America 6, 1083-1092.

Bovens, L. and Hartmann, S. (2000) 'Coherence, Belief Expansion and Bayesian Networks', in C. Baral and M. Truszczynski (eds) Proceedings of the 8th International Workshop on Non-Monotonic Reasoning, NMR 2000 (http://www.cs.engr.uky.edu/nmr2000/proceedings.html).

Bovens, L. and Hartmann, S. (forthcoming) 'Solving the Riddle of Coherence', Preprint Boulder and Konstanz.

Brown, L. (ed.) (1993) Renormalization: From Lorentz to Landau (and Beyond) (New York: Springer).

Bunge, M. (1964) 'Phenomenological Theories', in M. Bunge (ed.) The Critical Approach to Science and Philosophy. In Honor of Karl R. Popper (London: Collier-Macmillan), pp. 234-254.

Cao, T.Y. (1993) 'New Philosophy of Renormalization: From the Renormalization Group Equations to Effective Field Theories', in Brown (ed.) (1993), pp. 87134.

Cao, T.Y. (1997) Conceptual Developments of 20th Century Field Theories (Cambridge: Cambridge University Press).

Cao, T.Y. (ed.) (1999) Conceptual Foundations of Quantum Field Theory (Cambridge: Cambridge University Press).

Cao, T.Y. and Schweber, S. (1993) 'The Conceptual Foundations and the Philosophical Aspects of Renormalization Theory', Synthese 97, 33-108. 
Cassidy, D. (1995) Werner Heisenberg. Leben und Werk (Heidelberg: Spektrum Akademischer Verlag).

Cat, J. (1998) 'The Physicist's Debate on Unification in Physics at the End of the 20th Century', Historical Studies in the Physical and Biological Sciences 28, 253-299.

Cartwright, N. (1999) The Dappled World: A Study of the Boundaries of Science (Cambridge: Cambridge University Press).

Dirac, P (1983) 'The Origin of Quantum Field Theory', in L. Brown and L. Hoddeson (eds), The Birth of Particle Physics (Cambridge: Cambridge University Press), pp. 39-55.

Donoghue, J., Golowich, E. and Holstein, B. (1992) Dynamics of the Standard Model (Cambridge: Cambridge University Press).

Donoghue, J. (1994a) 'Leading Quantum Corrections to the Newtonian Potential', Physical Review Letters 72, 2996-2999.

Donoghue, J. (1994b) 'General Relativity as an Effective Field Theory: The Leading Quantum Corrections', Physical Review D59, 3874-3888.

Ecker, G. (1995) 'What is a Low-Energy-Theorem?', Comments on Nuclear and Particle Physics 21, 347-367.

Euler, H. (1936) 'Über die Streuung von Licht an Licht nach der Diracschen Theorie', Annalen der Physik 26, 398-448.

Fermi, E. (1933) 'Tentativo di una teoria del l'emissione dei raggi $\beta$ ', Ric. Sci. 4, 491-495.

Fermi, E. (1934) 'Versuch einer Theorie der $\beta$-Strahlen I', Zeitschrift für Physik 88, $161-171$.

Fischer, M. (1999) 'Renormalization Group Theory: Its Basis and Formulation in Statistical Physics', in Cao (1999), pp. 89-134.

Forster, M. (2000) 'Key Concepts in Model Selection: Performance and Generality', Journal of Mathematical Psychology 44, 205-231. 
Franklin, A. (1990) Experiment, Right or Wrong (Cambridge: Cambridge University Press).

Georgi, H. (1989) 'Effective Quantum Field Theories', in P. Davies (ed.), The New Physics (Cambridge: Cambridge University Press), pp. 446-457.

Georgi, H. (1993) 'Effective Field Theory', Annual Review of Nuclear and Particle Science 43, 209-252.

Hartmann, S. (1995a) Metaphysik und Methode. Strategien der zeitgenössischen Physik in wissenschaftsphilosophischer Perspektive (Konstanz: Hartung-Gorre Verlag).

Hartmann, S. (1995b) 'Models as a Tool for Theory Construction. Some Strategies of Preliminary Physics', in W. Herfel et al. (eds), Theories and Models in Scientific Processes (Amsterdam: Rodopi), pp. 49-67.

Hartmann, S. (1999) 'Models and Stories in Hadron Physics', in M. Morgan and M. Morrison (eds), Models as Mediators (Cambridge: Cambridge University Press), pp. 326-346.

Hartmann, S. (forthcoming) 'Review of S. French et al.: Correspondence, Invariance and Heuristics. Essays in Honour of Heinz Post, to appear in Studies in History and Philosophy of Modern Physics.

Heisenberg, W. (1966) 'Die Rolle der phänomenologischen Theorien im System der theoretischen Physik', in A. De-Shalit, H. Feshbach and L. Van Hove (eds), Preludes in Theoretical Physics. In Honor of V. F. Weisskopf (Amsterdam: North-Holland), pp. 166-169.

Heisenberg, W. and Euler, H. (1936) 'Folgerungen aus der Diracschen Theorie des Positrons', Zeitschrift für Physik 98, 714-732.

Huggett, N. and Weingard, R. (1995) 'The Renormalization Group and Effective Field Theories', Synthese 102, 171-194.

Humphreys, P. (1994) 'Numerical Experimentation', in P. Humphreys (ed.), Patrick Suppes: Scientific Philosopher, Vol. 2 (Dordrecht: Kluwer Academic Publishers), pp. 103-121.

Humphreys, P. (1995) 'Computational Science and Scientific Method', Minds and Machines 5, 499-512. 
Itzykson, C. and Zuber, J. B. (1980) Quantum Field Theory (New York: McGrawHill).

Jauch, J. and Rohrlich, F. (1976) The Theory of Photons and Electrons (Berlin: Springer).

Kitcher, P. (1989) 'Explanatory Unification and the Causal Structure of the World', in P. Kitcher and W. Salmon (eds), Scientific Explanation (Minneapolis: University of Minnesota Press), pp. 410-505.

Kolck, U. van (1999) 'Effective Field Theory of Nuclear Forces', Progress in Particle and Nuclear Physics 43, 337-418.

Kragh, H. (1990) Dirac. A Scientific Biography (Cambridge: Cambridge Uiversity Press).

Lepage, P. (1989) 'What is Renormalization?', in T. DeGrand and D. Toussaint (eds), From Actions to Answers, Proceedings of the 1989 Theoretical Study Institute in Elementary Particle Physics (Singapore: World Scientific), pp. 483509.

Lepage, P. (1994) 'Lattice QCD for Small Computers', Lectures presented at TASI 93 (Boulder, June 1993), and at the UK Summer Institute (St. Andrews, August 1993), hep-lat/9403018.

Leutwyler, H. (1994) 'Foundations and Scope of Chiral Perturbation Theory', Talk given at the conference 'Chiral Dynamics: Theory and Experiment' (MIT, July 1994), Preprint BUTP-94/18.

Meissner, U.-G. (ed.) (1992) Effective Field Theories of the Standard Model (Singapore: World Scientific).

Migdal, A. (1977) Qualitative Methods in Quantum Theory (London: Benjamin).

Mills, R. (1993) 'Tutorial on Infinities in QED', in Brown (1993), pp. 59-86.

Montvay, I. and Münster, G. (1994) Quantum Fields on a Lattice (Cambridge: Cambridge University Press).

Mosel, U. (1999) Fields, Symmetries, and Quarks (Springer: Berlin). 
Redhead, M. (1999) 'Quantum Field Theory and the Philosopher', in Cao (1999), pp. 34-40.

Robinson, D. (1992) 'Renormalization and the Effective Field Theory Programme', in D. Hull, M. Forbes, and K. Okruhlik (eds), PSA 1992, Vol. 1 (East Lansing: The Philosophy of Science Association), pp. 393-403.

Rohrlich, F. (1988) 'Pluralistic Ontology and Theory Reduction in Physical Sciences', British Journal for the Philosophy of Science 39, 295-312.

Rohrlich, F. (2000) 'Cognitive Scientific Realism' Philosophy of Science 67 (forthcoming).

Salmon, W. (1989) Four Decades of Scientific Explanation (Minneapolis: University of Minnesota Press). Reprinted in P. Kitcher and W. Salmon (eds) (1989), Scientific Explanation (Minneapolis: University of Minnesota Press), pp. 3219.

Salmon, W. (1998) Causality and Explanation (Oxford: Oxford University Press).

Schweber, S. (1993a) 'Changing Concepualization of Renormalization Theory', in Brown (ed.) (1993), pp. 135-166.

Schweber, S. (1993b) 'Physics, Community and the Crisis in Physical Theory', Physics Today 46, 34-40.

Schweber, S. (1994) QED and the Men Who Made It: Dyson, Feynman, Schwinger, and Tomonaga (Princeton: Princeton University Press).

Schweber, S. (1995) 'Physics, Community and the Crisis in Physical Theory', in K. Gavroglu (ed.), Physics, Philosophy and the Scientific Community (Dordrecht: Kluwer), pp. 125-152.

Schwinger, J. (1973) Particles, Sources, and Fields (Reading, MA: Addison-Wesley).

Shankar, R. (1999) 'Effective Field Theory in Condensed Matter Physics', in Cao (1999), pp. 47-55.

Weinberg, S. (1979) 'Phenomenological Lagrangians', Physica 96A, 327-340.

Weinberg, S. (1980a) 'Conceptual Foundations of the Unified Theory of Weak and Electromagnetic Interactions', Reviews of Modern Physics 52, 515-523. 
Weinberg, S. (1980b) 'Effective Gauge Theories', Physics Letters B91, 51-55.

Weinberg, S. (1987) 'Newtonianism, Reductionism, and the Art of Congressinal Testimony', Nature 330, 433-437.

Weinberg, S. (1993) Dreams of a Final Theory (London: Hutchinson Radius).

Wightman, A. (1986) 'Some Lessons of Renormalization Theory', in J. de Boer, E. Dal and O. Ulfbeck (eds), The Lessons of Quantum Theory (Amsterdam, Elsevier), pp. 201-226. 